\title{
Duodenal Perforation Caused by Distal Migration of Biliary Stent
}

\author{
Benjamin Y. M. Tan'1, George P. C. Yang2 \\ ${ }^{1}$ Loma Linda University School of Medicine, Loma Linda, USA \\ ${ }^{2}$ Department of Surgery, Hong Kong Adventist Hospital, Hong Kong, China \\ Email: ybtan@llu.edu, geroge.yang@hkah.org.hk
}

Received 9 March 2016; accepted 23 April 2016; published 26 April 2016

Copyright (C) 2016 by authors and Scientific Research Publishing Inc.

This work is licensed under the Creative Commons Attribution International License (CC BY). http://creativecommons.org/licenses/by/4.0/

(c) () Open Access

\begin{abstract}
Background: Migration of endoscopically placed biliary stents is a known complication of endoscopic retrograde cholangiopancreatography, which has a reported incidence of $3 \%-6 \%$. Usually, distal migration is asymptomatic and is detected incidentally. Case Presentation: We present a rare case of duodenal perforation caused by distal migration of a biliary stent. A 50 years old gentleman initially presented with cholangitis. ERCP showed a dilated common bile duct with a $9 \mathrm{~mm}$ stone and a biliary stent was placed. The patient subsequently presented with biliary peritonitis due to the migration of the stent causing perforation of the anterior wall of the second part of the duodenum. Emergency laparotomy was performed to remove the stent and the perforation was repaired. The patient made an uneventful recovery. Discussion: A brief discussion on stent migration is also conducted. Whilst distal migration of stents is less common than its proximal counterpart, it is important to note that rare complications may arise and be addressed in a timely manner.
\end{abstract}

\section{Keywords}

Cholangiopancreatography, Endoscopic Retrograde, Cholangitis, Intestinal Perforation

\section{Introduction}

Endoscopic biliary stent insertion is a well-established treatment of choice for relieving obstructive jaundice in both benign diseases such as choledocholithiasis as well as unresectable hepatobiliary malignancy. However, it is not without complications which range between $8 \%$ to $10 \%$ [1]. Biliary stent specific complications include migration, occlusion and intestinal obstruction. In the majority of instances, stent migration is asymptomatic. Nevertheless, there are several sporadic reports of small bowel perforation, colonic diverticular perforation, co- 
lonic fistula and intra-abdominal abscess caused by biliary stent migrations in literature [2] [3]. Herein we present a case of duodenal perforation caused by the distal migration of a biliary stent. A brief discussion of proposed mechanism is also conducted.

\section{Case Presentation}

A 50 years old gentleman was admitted to our hospital for cholangitis. He presented with fever and right upper quadrant pain. Blood tests showed leukocytosis and deranged liver function in an obstructive pattern. Urgent ultrasonography of abdomen confirmed a dilated biliary tree and the presence of gallstones. Emergency endoscopic retrograde cholangiopancreatography (ERCP) was done which showed a dilated common bile duct (CBD) to $15 \mathrm{~mm}$ with a $9 \mathrm{~mm}$ stone in the distal common bile duct. A 10 French, $10 \mathrm{~cm}$ long plastic biliary stent was inserted for biliary drainage. He was discharged a few days later uneventfully and was planned for ERCP and removal of stones as well as interval cholecystectomy later.

However, he was readmitted 1 week after ERCP, complaining of severe abdominal pain. Abdominal examination revealed guarding at upper abdomen guarding at upper abdomen with rebound tenderness. The patient was tachycardic with a pulse of $140 / \mathrm{min}$. His respiratory rate was $20 / \mathrm{min}$. Laboratory investigations showed a white cell count of $23 \times 10 \mathrm{e} / \mathrm{L}$ and a bilirubin of $32 \mathrm{umol} / \mathrm{L}$. Hemoglobin, ALP, ALT, AST, amylase and renal function tests were within normal limits. CXR showed free gas under the diaphragm.

Urgent abdominal computer tomography (CT) scan confirmed the presence of free gas and distal migration of the biliary stent causing perforation of the duodenum. Emergency laparotomy showed biliary peritonitis and the plastic stent was seen protruding from the anterior wall of the second part of the duodenum (Figure 1). The stent was removed, exploration of the common bile duct was performed together with a T-tube placement. The duodenal perforation was repaired, the perforation site was closed with an omental patch and an abdominal drain was placed next to the duodenal perforation.

The patient was put on total parental nutrition post-operatively for 3 weeks. A T-tube cholangiogram, performed two weeks post-operatively showed no leakage from the duodenum, with free passage of contrast down into the distal small bowel. The T-tube was clamped and the patient started on fluids and subsequently on a solid diet. The abdominal drain was removed 4 days after the patient resumed oral feeding. He was discharged 4 weeks after admission and the T-tube was removed 2 months post-operatively. Interval cholecystectomy was subsequently performed. The patient was followed up for two years during which time he had no further abdominal complaints.

\section{Discussion}

Endoscopic biliary stent insertion has been used for many years as a temporary or definitive treatment for biliary obstruction. Two commonly available stents are classified according to synthetic material, namely plastic and

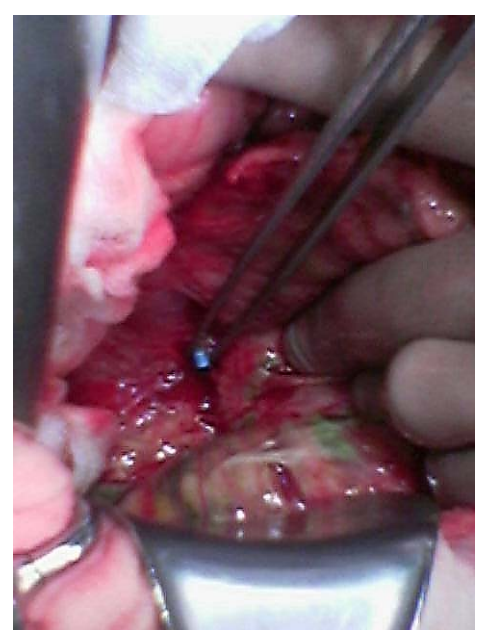

Figure 1. Biliary stent protruded out from the anterior wall of second part of duodenum. 
metallic stents. The majority of plastic stents are straight type with a slightly curved shaft and flaps near both ends to prevent proximal and distal migration. According to a retrospective review studying 378 biliary plastic stent insertions, $13.5 \%$ presented with either proximal or distal migration; $7.9 \%$ for proximal and $5.9 \%$ for distal migration respectively. All the patients with proximally migrated stent presented with cholangitis or recurrence of painless jaundice which required endoscopic retrieval of stents, while distally migrated stent patients were usually asymptomatic, and presented as an incidental finding on follow up ERCP [4].

Despite the relatively innocent behavior of distal stent migration, there are numerous rare complications reported in the literature, such as duodenal perforation, colonic perforation resulting in pelvic abscess, enterocutaneous fistula or necrotizing fasciitis. Distal stent migration more commonly occurs in benign compared to malignant strictures. This is because benign stenosis would undergo regression once the inflammatory reaction has subsided like what has occurred in our patient, while malignant stenosis may grow further and can tightly anchor the stent in position. Other important risk factors for distal stent migration include inappropriate stent selection and stent placement. In other words, if too long a stent was chosen, or if there was malposition of stent with a long segment within the duodenal lumen. This could result in impaction of the duodenal wall and perforation of duodenum. Perforation of the duodenum can be intra-peritoneal resulting in biliary peritonitis or retro-peritoneal causing a bilioma [5]. Besides the duodenum, other common sites of perforation are the diverticular sac of the large bowel, as the walls of the diverticula are extremely thin, or in fixed areas of the intestines because of adhesion from previous operations.

The treatment for distal stent migration is quite controversial. In cases of obvious peritonitis and unstable hemodynamics, immediate surgical exploration is warranted. In the literature, there was also reporting of endoscopic extraction of migrating stent and endoscopic clip closure of duodenal perforation [6]. In the case of retroperitoneal perforation and localized bilioma, successful percutaneous extraction of stent and drainage has been reported [7].

\section{Conclusion}

To conclude, distal stent migration is not innocent; it can cause potentially lethal complications as seen in our presented case. In order to prevent such complications from occurring, endoscopists should choose the correct size, length and shape of the biliary stent, and avoid prolonged placement of stent in benign biliary obstruction.

\section{References}

[1] Mueller, P.R., Ferrucci, J.T., Teplick, S.K., et al. (1985) Biliary Stent Endoprosthesis: Analysis of Complications in 113 Patients. Radiology, 156, 637-639. http://dx.doi.org/10.1148/radiology.156.3.4023221

[2] Bharathi, R.S., Rao, P.P. and Ghosh, K. (2008) Intra-Peritoneal Duodenal Perforation Caused by Delayed Migration of Endobiliary Stent: A Case Report. International Journal of Surgery, 6, 478-480. http://dx.doi.org/10.1016/j.ijsu.2006.06.012

[3] Namdar, T., Raffel, A.M., Topp, S.A., et al. (2007) Complications and Treatment of Migrated Biliary Endoprosthesis: A Review of the Literature. World Journal of Gastroenterology, 13, 5397-5399. http://dx.doi.org/10.3748/wjg.v13.i40.5397

[4] Katsinelos, P., Kountouras, J., Paroutoglou, G., Chatzimavroudis, G. and Paikos, D. (2009) Migration of Plastic Biliary Stents and Endoscopic Retrieval: An Experience of Three Referral Centers. Surgical Laparoscopy, Endoscopy \& Percutaneous Techniques, 3, 217-221. http://dx.doi.org/10.1097/SLE.0b013e3181a031f5

[5] Miller, G., Yim, D., Macari, M., Harris, M. and Shamamian, P. (2005) Retroperitoneal Perforation of the Duodenum from Biliary Stent Erosion. Current Surgery, 62, 512-515. http://dx.doi.org/10.1016/j.cursur.2005.03.011

[6] Rosés, L.L., Ramirez, A.G., Seco, A.L., et al. (2000) Clip Closure of Duodenal Perforation Secondary to a Biliary Stent. Gastrointestinal Endoscopy, 51, 487-489. http://dx.doi.org/10.1016/S0016-5107(00)70454-8

[7] Bui, B.T., Oliva, V.L., Ghattas, G., Daloze, P., Bourdon, F. and Carignan, L. (1995) Percutaneous Removal of a Biliary Stent after Acute Spontaneous Duodenal Perforation. CardioVascular and Interventional Radiology, 18, $200-202$. http://dx.doi.org/10.1007/BF00204152 